\title{
Variaciones geográficas de aislamiento de aeromonas hydrophila en niños con diarrea aguda infantil y población sana
}

\author{
Dra, Olivie Trueco A. ${ }^{1}$; Dra. Valcria Prado J. ${ }^{2}$ : Drd. M. Teresa Siri A. ${ }^{2}$; \\ Dra. Gloria Dib C. ${ }^{3}$; Dr. Vicente Millán T. ${ }^{3}$; T.M. Juan Martínez D. ${ }^{2}$; \\ T.M. Lucia Reyes M. ${ }^{2}$; T.M. Nora Maniani M. ${ }^{2}$

\section{Geographic differences in isolation of aeromonas hydrophila from infants with and without acute diarrhea}

\begin{abstract}
Aeromonas hydrophila has been recently described as ethiological agent of acute diarrhea. In order to determine its role as potential enteropathogen in clujean infants we studied 285 infants and todlers ( 1 to 24 months old) with acute diarthea and 140 controls matched by age, sex and sociocconomic level, from two cities (Santiugo and Valparaíso) during the summer periods of $1983 / 84$ and $1985 / 86$. In the Valparaiso group, jsolation rates of Aeromonas hydrophila were $5.5 \%$ in infants with acute diarrhea and $2.3 \%$ in controls $(p<0.05$ ). Among infants from Santiago, no A. hydrophila strains were isolated. Enter otoxin production was negative, (both ST and I,T) in all the A. hydrophila strains. The geograplic differences in isolation rates of Aeromonas may be relited to the presence of acuatic reservoirs.
\end{abstract}

(Key words: Acromonas hydrophila, acute dijurlyca, inlants, geographic differences).

Desde el primer aislamiento de Aeromonas en deposiciones por Lantrop en 1961, en una epidemia de diarrea aguda, se ha intentado determinar el rol de este potencial patógeno intestinal tanto en niños como en adultos. Han sido múltiples las comuricaciones en las últimas dos décadas que involucran a Aeromonas en cuadros de gastroenteritis $^{1-7}$.

1. Becado programa formación especialista en microbiología. Facultad de Medicina Universidad de Chile.

2. Unidad Microbiología. Depto. Salud Pública. Div. Cs. Médicas Oriente, Facultad de Medicina, Universidad de Chile.

3. Servicio Pediatría. Hospital Deformes, Valparaíso.
Se sabe que el reservorio natural de esta bacteria es fundamentalmente acuático (aguas saladas y dulces) encontrándose también en suelos y en flora intestinal de individuos sanos, en forma transitoria aunque su incidencia no ha sido bien establecida ${ }^{3,9,10}$.

La actual clasificación del géneto Aeromonas incluye 3 especies móviles (A. cariae, A. hydro. phila, A. sobria) y una especie innóvil (A. sal. monicida) esta última no asociada con patología humana. A. hydrophila ha sido la más comúnmente aislada en cuadros de gastroenteritis 11,12 .

A la dificultad para aclarar la participación de este agente en diarrea aguda, ha contribuido el 
hecho que estos organismos pueden ser fácilmentc confundidos con ciertos miembros de la familia Enterobacteriaceae en medios de cultivo corrientes. Es necesario incluir en el cstudio de coprocultivos, medio de cultivo selectivo para su desarrollo junto a la práctica corriente de prueba de oxidasa $13,14,15$.

Algunos hechos orientan a considerar a $A$. hydrophita como enteropatógeno: la prevalencia más elevada en pacientes con diarrea aguda en relación a controles que hin mostrado algunos estudios $^{12}$ la determinación de algunos factores de enteropatogenicidad y la respuesta humoral frente a la infección. Se asocian con mayor frecuencia a cuadros de gastroenteritis, cepas enterotoxigénicas siendo ta! vez este mecanismo de patogenicidad el más importante. También se han descrito numerusos fuctores extracelulares asociados con vitulencia bacteriana como hemolisinas, proteasas, citotoxinas, fimbrias y otras $^{16 \cdot 18}$.

Ya que cl sindrome diarreico agudo continúa siendo un problema frecuente en nuestra población infantil, para obtener mayor información acerea de la epidemiología, incidencia y patogenicidad de esta bacteria, nuestro grupo realizó uria búsqueda sistemática de Aeromonas en niños menores de 2 años con diarrea aguda y controles en 2 periodos de verano $1983-1984$ y $1985-86$, estudiando pacientes de 2 ciudades on diferentes caracter isticas ecológicas en lo que se refiere a reservorios naturales de aguz.

\section{MATERIAL $Y$ METODOS}

Se estudiaron 285 niños menores de 2 años de nivel socioeconómico medjo bajo ton síndrome diarreico agudo y 140 niños controles parcados por edad y sexo, durante los veranos 1983-84 y 85-B6. Diarrea lue definida como cuadro clínico earacterizado por deposiciones de mayor contenido acuoso que lo normal con o sin clementos patológicos (mucus, pus y/o singre) con inás de 3 eracuaciones en el día, duración más de un dia y menos de 7 dias.

Del grupo con diartea aguda, 73 niños eran del hospital Deformes de Valparaiso y 212 del hospital Luis Calvo Mackesuna de Santiago y del consultorio La Faena (Ared de Salud Metropolitano Oriente).

Entrc los grupos controles 44 niños eran de Valpataiso y 96 niños de Santiago (consultorio La Ficna).

Sc tomó una muestra de deposición recién emitida o por hisopado rcetal a cada niño, Ias muestras de Valparaike fueron transportadas en medio Cary Blair. $\$$ confeccionó un cuestionario clínico-epidemiológico en cada caso.

Las muestras fueron sembradas en agat SS, agar Mac Conkey, agar Campy Bap, agar selcctivo para Yersinia $y$ agat sangre con ampicilina. La identificación de los diferentes enteropatógenos se efectuó de acuerdo a recomendaciones inter nacionales $\mathbf{1 9 - 2 3}$.

Lus muestras de deposiciones fueron sembradas en agar sungre base más $5 \%$ de sangre de cordero adjcionado de $15 \mu \mathrm{g} / \mathrm{ml}$ de ampicilisa 14,15 . A las oolonias de color griśaceo con o sin betahemólisis se les realizó prueba de oxidasil $y$ bater ía de medios diferenciales para tlegar a diagnóstico de Género, la confirmación y tipiIieación definitiva fue hecha por el Instituto de Salud Públicis (ISP).

Sc efectuó estudio de enter otoxinas, termolábil (LT) $y$ termoestable (ST) en todas las cepas de A eromonas hydrophilo aisladas; toxina LT mediante técnica de T:LISA utilizando sobrenadante de cultivo y colonias aislados paralelanente ${ }^{24}$. Lu búsqueda de toxina ST se realizó mediante inoculación intragástrica en ratón lactante (técnica de Dean) 23 .

Fi. estudio de sensibilidad a los antimicrobianos, de las Acromonas aj sladas scectuó mediante la lécnica de dilutión en placa deter ninando conentración inhibitoria mínitna (CJM) de los siguientes antimicrobianos: cloramfenicol, cotrimoxazol, gentamicina, amikacina, dibekacina, efotaxima, ceftriaxone, cefoperazona siguiendo la téenica internacional recomendada. Los valores de corte para considerar scnsibles a las ecpas estudiadas fueron: closamfenicol $=12.5$; cotrinoxazol $=80$ : gentamicina $=4$; amikacina $=16$; dibekacina $=4$; wotaxima $=16$; ceftriaxona $=16$; weperazona $=16^{25}$

\section{RESULTADOS}

Los resultados de los dos períodos de estudio (verano 1983.84 y 85.86 ) dieron un aislamiento global de Aeromonas hydrophila de 5,5\% entre lactantes con diarrea aguda en Valparaiso y $2,3 \%$ entre controles de esa misma ciudad; diferencia estadisticamente no significativa $(\mathrm{p}<0,05)$. En el grupo de niños de Santiago no se aislaron Aeromonas ni en pacientes ni en controles (Tabla 1).

La Tabla 2 analiza la importancia relativa que tiene Aeromonas entre los agentes de gastroenteritis en población menor de 2 affos, ocupando un lugar destacado entre los niños de Valparaíso, no participando como enteropatógeno en Santiago.

Los resultados del estudio de sensibilidad de las cepas de Acromonas hydrophila aisladas se observa en la Tabla 3, la que muestra dos cepas resistentes a cloramfenicol (CIM $\geqslant 12,5 \mu \mathrm{g} / \mathrm{ml}$ ); e) resto de los antimicrobianos estudiados fueron

Tabta 1.

Frecuencia poroentual de Aeromonas hydrophila aisladas en lactantes de Santiago y Valparaíso. Veranos $1983 / 84$ y $85 / 86$

\begin{tabular}{|l|cc|}
\hline & $\begin{array}{c}\text { Recuperación Aeromonas hudrophila } \\
\text { Santiago } \\
N^{\circ} / N^{0} \text { total }\end{array}$ & $\begin{array}{c}\text { Valparaíso } \\
N^{\circ} / N^{0} \text { total }\end{array}$ \\
Diarrea aguda & $0 / 212$ & $4^{*} / 73(5,5 \%)$ \\
Controles & $0 / 96$ & $1^{*} / 44(2,3 \%)$ \\
\hline TOTAL & $0 / 308$ & $5 / 117$ \\
\hline
\end{tabular}

* único enteropa tógeno. 
Tabla 2.

Importancia relativa de Acromonas respecto de otras bacterias entcropatógenas en lactantes de San1iago y Valparaíso. Veranss 1983/84 y 85/86

\begin{tabular}{|c|c|c|c|c|c|}
\hline \multirow[b]{2}{*}{ Bacter ias entcropatógenas } & \multicolumn{2}{|c|}{ SANTIACO } & \multicolumn{3}{|c|}{ VALPARAISO } \\
\hline & $\begin{array}{l}\text { Diarrea } \\
\mathrm{n}=212\end{array}$ & $\begin{array}{c}\text { Control } \\
n=96\end{array}$ & $\begin{array}{c}\text { Diarrea } \\
\mathrm{n}=73\end{array}$ & $\begin{array}{c}\text { Control } \\
n=44\end{array}$ & $\mathbf{P}$ \\
\hline & $\%$ & $\%$ & $\%$ & $\%$ & \\
\hline E. coli enteropatógeno & 34,9 & 14.6 & 26 & 11,4 & \\
\hline 1. coli enterotoxigénico* & 19 & 9 & ne & ne & \\
\hline E. coli enteroinvasor & 1,4 & 1 & 0 & 0 & \\
\hline Shigella & 10.8 & 0 & 2,7 & 0 & \\
\hline Campylobacter & 6,1 & 0 & 2,7 & 0 & \\
\hline Aeromonas & 0 & 0 & 5,5 & 2,3 & $\geqslant 0,05$ \\
\hline Salmonella & 0,9 & 0 & 0 & 0 & \\
\hline Yersinia & 0 & 0 & 0 & 0 & \\
\hline
\end{tabular}

* ECET corresponde a 161 pacientes con diarrea y 74 controles. ne $=$ no estudiado.

Tabla 3.

Actividad comparativa in vitro (CIM acg/ml) de 8 antimicrobianos frente a 5 cepas de Aeromonas hydrophila

\begin{tabular}{|c|c|c|c|c|c|}
\hline \multirow{2}{*}{ Antimicrobiano } & \multicolumn{5}{|c|}{$\operatorname{CIM}(u c g / n l)$} \\
\hline & 1 & 2 & 3 & 4 & 5 (cepas) \\
\hline Cloramfenicol & 0,5 & 256 & 256 & 8 & 2 \\
\hline Cotrimoxazol & 2 & 2 & I & $t$ & \\
\hline Gentamicima & $\leqslant 0,25$ & 0,5 & 0,5 & $\leqslant 0,25$ & $\leqslant 0,25$ \\
\hline A mikaciru & 2 & 2 & 2 & 2 & 1 \\
\hline Dibekacina & 1 & 1 & 1 & 0.5 & 0,5 \\
\hline Cefotaxima & $\leqslant 0,25$ & $\leqslant 0,25$ & $\leqslant 0,25$ & $\leqslant 0.25$ & $\leqslant 0,25$ \\
\hline Ceftriaxoura & $\leqslant 0,25$ & $\leqslant 0,25$ & $\leqslant 0,25$ & $\leqslant 0,25$ & $\leqslant 0,25$ \\
\hline Cefoperazond & $\leqslant 0,25$ & 8 & 2 & 1 & 2 \\
\hline
\end{tabular}

activos frente a la totalidad de las cepas. $\mathbf{L a}$ mayor actividad in vitro correspondió a Ceftriaxone y Cefotaxima.

En el estudio de cnterotoxicidad de las 5 cepas de Aeromonas hydrophila. la búsqueda de toxina termolábil (LT) resultó negatjya en todas mediante técnica de ELISA, tanto en sobrenadantes de cultivo como con cultivg directo de colonia. Tampoco se detectó producción de toxina termoestable (ST) en ninguna de estas cepas, en el modelo de ratón lactante.

\section{COMENTARIO}

Los resultados de este estudio nos estarían indicando que Aeromonas hydrophila parece tener una distribución regiona] variable en nuestro medio. Se aisló Aeromonaś en deposiciones sólo de niños que provenían de Valparaíso no aislándose este agente en Santiago, variación que tal vez podría explicarse por diferencias ecológj- cas en relación a la existencia de reservorios naturales de este potencial enteropatógeno.

Resultó ser más frecuente su aislamiento entre niños con diarrea aguda que en controles, diferencia que no resultó estadisticamente significariva. Estos datos concuerdan con otros autores como Pitarangsi ${ }^{6}$ y Gracey ${ }^{1}$ quienes no encon. traron diferencias significativas cuando se trataba de cepas no enterotoxigénicas, situación que ocurrió en nuestro estudio ya que en ninguna de las cepas de Aeromonas hydrophila se demostró la presencia de enterotoxina ST ni LT

Todas las cepas aisladas correspondieron a la especie $A$. hydrophila, que ba sido la más frecuentemente involucrada como agente de gastroenteritis, sin embargo se han descrito cuadros similares al cólera producidos por $A$. sobria ${ }^{3}$.

Según lo descrito, la hemolisis se relaciona con capacidad enterotóxica en $96 \%$ a más de los casos, lo que tambiến resultó concordante con nuestras observaciones, ya que las 5 cepas de 
Aeromonas hydrophila aisladas no producian hemolisis en agar sangre ni eran enterotoxigénicas.

Respecto de las características clínicas de cuadros de gastroenteritis en los cuales se ha ajslado Aeromonas como único agente enteropatógeno, se han observado diferentes perfjles clínj$\cos$, desde diarreas leves de corta duración a síndrome disentéricos graves y también gastroenteritis con deposiciones líquidas profusas similares al cólera. En nuestra experiencia los pacientes en que se aisló Aeromonas, 4 presentaron diarrea aguda con deshidratación leve, las deposiciones fueron descritas como líquidas de frecuencia entre 4 a 6 por dia, sin elementos patológicos (pus o sangre).

No presentaron fiebre ni vómitos y el estado nutricional de los niños al ingreso al hospital era bueno (eutróficos). Sólo uno de tos njn̄os habia recibido medicamentos previo al estudio (antiparasitario). El otro niño en que se aisló Aeromonas estaba asintomático y pertenecía al grupo control.

La frecuencia de portadores ha sido variable en diferentes estudios dependiendo del empleo de medios de cultivo selectivos que facilitan el aislamiento de $A$. hydrophila; Graevenitz en 1968 reportaba $0,7 \%$ de portación subiendo a $3,2 \%$ en estudios posteriores al introducir agar con ampicilina ${ }^{5}$, Millership cncontró $4,2 \% \%^{8}$, valores similares a la frecuencia de $2,3 \%$ detectada en nuestro estudio.

Se han publicado muchos estudios relacionados con sensibilidad antimicrobiana y hasta la fecha tho se han descrito cepas de $A$. hydrophilo sensibles a ampicilina, de ahí que este antimicrobiano sea útil como agente selectivo en medios de aisíamiento. Sin embargo, se han descrito cepas de $A$. sobria sensibles a ampicilina, especie que también ha sido involucrada en diarrea aguda aunque con menor frecuencia. En general las cepas aisladas en gastroenteritis se describen como sensibles a gentamicina, cloramfenicol. ceftazidima $y$ otros apareciendo resistencia a eritromicina, clindamicina, cefradina, cefazo. lina ${ }^{26}$. En nuestro estudio encontramos 2 cepas resistentes a cloramfenicol siendo sensibles al resto de los antimicrobianos estudiados.

Es interesante destacar los diferentes elementos asociados con virulencia bacteriana que se han descrito en $A$. hydrophila: fimbrias de adhe. rencia que iniciarían el proceso infeccioso, citotoxinas las cuales han sido estudiadas en diferentes líneas celulares (fibroblastos humanos, células tumorales humanas: HELA, células de riñón de mono verde africano), enterotoxinas y una gran variedad de enzimas entre ellas hemolisinas, proteasas, lecitinasas, nucleasas, amilasas, elastasas $y$ otras.

$S$ in embargo es necesario acumular nayor información tanto epiđemiológica como patogénica, para definir mejor la importancia de $A$. hydrophila en el sindrome diarreico agudo.

\section{RESUMEN}

Entre los nuevos agentes capaces de producir diarrea aguda se ha descrito la participación de Aeromonas hydrophila. Con objeto de conocer la incidencia e importancia relativa de este puten. cial enteropatógeno en nuestro medio, estudiamos 285 niños menores de 2 años con sindrome diarreico agudo y 140 niños controles pareados por edad y sexo en 2 ciudades (Santiago y Valparaíso) durante los veranos 1983-84 y 85-86.

El aislamiento de Aeromonas hydrophila en pacientes de Valparaíso fue de $5,5 \%(4 / 73)$ en niños con diarrea y $2,3 \%(1 / 44)$ en controles, diferencia estadisticamente no significativa. No hubo aislamiento de este agente en Santiago (0/212). Se efectuó estudio de enterotoxicjdad de las cepas aisladas que resultó negativo tanto para enterotoxinas termoestable (ST) mediante modelo de tatón lactante y para enterotoxina termolábil (LT) mediante ELISA.

Las variaciones geograficas observadas en el aislamiento de Aeromonas en diarrea aguda, puede correlacionarse tal vez con existencia de reservorios acuáticos naturales.

\section{REFERENCIAS}

1. Gracey, M., Bruke, $\gamma_{.}$, Robinson, J.: Acromonas associa ted gastroenteritis. Lancet 2: 1304, 1982.

2. Lance, G., Nakata. M., Thompson, J., White, M.: Aeromonas related diarrhea in adults. Arch Intern Med 145: 2207, 1985.

3. Arampsam, A., Rothman, E., Mathieu, D., Andremont, A., Auzepy. P.: Choleralike illness due to Aesomonas sobria. J Infect Dis $145(2): 248$. 1982.

4. Morsik, F:: Other gram negative bacilli. Mandell, Douglas, Bennett II. Principles and Practice of Infectious Diseases. New York John Wiley \& Sons 1985 Second edition, 1318.

5. Graevenitz, A.: Acromonas y Plesiomonas. Lennette F. Manual of clinical Microbiology Washington. American Socicty for Micsobiology. Thitd Edition, 1980, 220-225.

6. Pitarantest, C., Echeverria, $P$. Whitmire, $R$ et al: Enteropathogenicity of Aeromonas hydrophila and Plesiomonas Shigelloides: Prevalence among individuals with and without diarrhea in Thailand. Infec Immun 35: 666, 1982 .

7. Davis, W.A., Kane, J., Garagush, V.: Human Aeromonas infections. Medicine, 57: 267, 1978.

8. Millership, S, Curnow, S: Clattopadhyay B. Faecal carriage rate of Aeromonas hydrophila. I Clin Pathol 36: 920, 1983. 
9. Gracey and colleagnes: Isolation of Aeromonas from faeces. Lancet 1: 35 I, 1983.

10. Shread, P.T., Donovan, $T$, Lee, J.: A survey of the incidence of Aeromonas in human faeces. Soc Gen Microbiol 8: 184, 1981 .

11. Agger, W.A. et al Clinical and microbiological features of Aeromonas hydrophita asuciated diarrheae. J Clin Micr obiol 21: 909, 1985.

12. Frey, B.J.: Aeromonas biology of the organism and diseases in children. Pediatr Infect Dis 3: 164, 1984.

13. Robinson, I., Bruke, V., Worthy, P.: Media for isolation of Aeromonas spp. from facces. J Med Microbiol 18: 405, 1984 .

14. Kay, B.A. ef al- Media for the isolation of Aeromonas hydrophila. J Clin Microbiol 22: 888, 1985.

15. Van Graevenitz, A. et ar: Evaluation of differential and selective media for isolation of Aeromonas and Plesiomonas from human feces. J Clin Microbiol 17: $16,1983$.

16. Honda, $P_{.:-}$Demonstration of cholera-toxin related factor in cultures of Aeromonas with FLISA. Infect Immun 50: 322, 1985.

17. Morgan, D.R.: Lack of correlation between known virulence properties of Aeromonas and enteropathogenicity for humans. Infect lmmun 50: 62, 1985 .

18. Cumberhatch, N., Gruwith, M.J, Laugton, $C$. Cytotoxic enterotoxin produced by A. hydrophila: relationship of toxigenic isolates to diarrheae discase. Infect Immun 23:829, 1979.

19. Edwards, P.R. Ewing, W.H.: Identificación of enterobacteriacea. 3o Ed. Burgess Publishing Co. Minneapolis, Minn. 1972.

20. OMS/OPS documento oficial 80,2.: $83^{\circ}$ Comité ejecutivo Programa de control de enfermedades diarreicas.

21. Sereny, B.: Experimental Shigella Keratoconjuntivitis: a preliminary report. $A$ cta Microbiol $\Lambda$ cad Sci 2: 293,1955

22. Honda, T., Glass, R., Akhtar, Q.: A simple assay to detect $E$. coli producing $L T$ enterotoxin: results of a field study of the Biken test in Bangladesh. Lancet 1: 609,1981 .

23. Dean, A,G., Ching, $Y$. et al: Test for $\mathrm{L}$. coli enterotoxin using infant mice: application in study of diarrheae in childsen in Honolulu. $J$ infect Dis 125: 407,1972 .

24. Ristaino, P.A., Levine, M.M., Young, Ch.R.: Improved $G M_{1}$-enzyme linked immunosorbent assay for detection of Escherichia coli heat-labile enterotoxin. J Clin Microbiol 18: 808, 1983

25. National Commitee for Clinical laboratory Standars, LSA. 1982.

26. Zemelman, $R$ et al: Aeromonas spp susioptibility to 22 antimicrobial agents Antimicrob. Agents Chemother. 28: 151, 1985 . 